\title{
The Enhanced Indispensability Argument, the Circularity Problem, and the Interpretability Strategy
}

\author{
Jan Heylen $\quad$ Lars Arthur Tump
}

\begin{abstract}
Within the context of the Quine-Putnam indispensability argument, one discussion about the status of mathematics is concerned with the 'Enhanced Indispensability Argument', which makes explicit in what way mathematics is supposed to be indispensable in science, namely explanatory. If there are genuine mathematical explanations of empirical phenomena, an argument for mathematical platonism could be extracted by using inference to the best explanation. The best explanation of the primeness of the life cycles of Periodical Cicadas is genuinely mathematical, according to Baker $(2005,2009)$. Furthermore, the result is then also used to strengthen the platonist position (e.g. Baker 2017a). We pick up the circularity problem brought up by Leng (2005) and Bangu (2008). We will argue that Baker's attempt to solve this problem fails, if Hume's Principle is analytic. We will also provide the opponent of the Enhanced Indispensability Argument with the so-called 'interpretability strategy', which can be used to come up with alternative explanations in case Hume's Principle is non-analytic.
\end{abstract}

Keywords indispensability; mathematical explanation; platonism; nominalism; Frege Arithmetic; Hume's Principle; relative interpretation

\section{Introduction}

The classical indispensability argument (Quine, 1948; Putnam, 1971) is the backdrop against which Baker (2005) has advanced the so-called 'Enhanced Indispensability Argument'. Baker (2005, p. 224) echoes a point made by Maddy (1992), to wit the fact that we do not tend to accept in our ontologies entities posited by our best scientific models such as 'frictionless slopes, ideal gases and infinitely-deep fluids'. Another important point, which Baker takes from an exchange between Melia (2000) and Colyvan (2002), is that we only ought to be ontologically committed to those entities that are indispensable in the right way, which according to them means indispensable for explanations. Common ground in this debate is that if there are mathematical entities that fulfill the same kind of explanatory role as theoretical entities in scientific theories, then mathematical entities are on par with theoretical entities and mathematical platonism prevails. Thus, the challenge is to find an example of such a mathematical 
explanation of a physical phenomenon. Baker (2005) purports to provide such an example. Furthermore, Baker (2017a) uses this example to further boost the cause for platonism.

In his (2005) paper, Baker presents a case in which he claims to have found an example, in evolutionary biology, of a mathematical explanation of a physical phenomenon. The example treats of the Periodical Cicada, which is a genus of the Cicada family, of which the species have either a 13- or a 17-year life cycle. One of the questions biologists are concerned with, is why the life cycles of these species are prime. For instance, whether it might be the case that this very fact would give them some evolutionary advantage. Thus, the thing to be explained, the explanandum, is the primeness of the life cycles of Periodical Cicadas. Baker gives the basic structure of the explanation (Baker, 2005, p. 233):

(1) Biological 'law':

Having a life cycle period which minimizes intersection with other periods is evolutionary advantageous.

(2) Number theoretic theorem:

Prime periods minimize intersection (compared to non-prime periods).

(3) 'Mixed' biological/mathematical law:

Hence organisms with periodic life cycles are likely to evolve periods that are prime.

(4) Ecological constraint:

Cicadas in ecosystem-type, E, are limited by biological constraints to periods from 14 to 18 years.

\section{(5) Species specific prediction:}

Hence cicadas in ecosystem-type, E, are likely to evolve 17-year periods.

In the context of indispensability arguments in general, and the Enhanced Indispensability Argument in particular, the step in the argument we are concerned with is $(2)^{1}$. As Field (1989, p. 15) puts it:

one of the assumptions that appears in this explanation is claim S, and we are pretty sure that no explanation of the phenomena that does without claim $\mathrm{S}$ is possible.

In our case, the reference to the number theoretic theorem in step (2) is S. It is an Enhanced Indispensability Argument, because the number theoretic theorem (2) has a genuine explanatory role, in a way quite different from for example idealized concrete objects (Baker, 2005, p. 237). The argument has generated a lot of discussion - see,

\footnotetext{
${ }^{1}$ In (2017a, pp. 783-784), Baker gives an alternative version of the argument. But the first three steps are the same as in the initial formulation. Baker points out that the length of the cicada life cycles can be expressed as sums of perfect squares, which introduces more sophisticated mathematics.
} 
for instance, (Rizza, 2011; Wakil and Justus, 2017; Baker, 2017b; Barrantes, 2019). In section 2 we pick up one problem that has been brought up, namely the circularity problem. Next, in section 3 we will explicate how Baker proposes to solve that problem. Subsequently, in section 4 we will argue that the problem shows up again once a gap, resulting from the attempted solution to the circularity problem, has been bridged by invoking Hume's Principle, if Hume's Principle is analytic. On the, nontrivial, assumption that Hume's Principle is not analytic, the defenders of the Enhanced Indispensability Argument have at their disposal a mathematical explanation based on Hume's Principle, without circularity. In response, in section 5 we will provide the opponent of the Enhanced Indispensability Argument with a strategy (called 'the interpretability strategy') to come up with an alternative explanation, thereby allowing for the assumption that Hume's Principle is not analytic, and challenging the further assumption that the Enhanced Indispensability Argument is indeed the best explanation. In line with the main concern of this paper, we point out that one criterion for the selection of the best explanation, namely mathematical power, is question-begging.

\section{The claim of circularity}

Analyzing the abovementioned example, Bangu notices that the explanandum, at least in the way it is presented as above,

consists in a property-attribution claim, where the property in question is primeness, a paradigmatic example of a mathematical property (Bangu, 2008, p. 18).

The problem with the fact that the explanandum contains a mathematical property is that, and we remember that the strategy of inference to the best explanation is accepted by both sides in the debate, the explanandum is assumed to be true. As Leng (2005, p. 174) puts it:

Given the form of [the] argument, one might wonder why it is mathematical explanations of physical phenomena that get priority. For if there are ... some genuine mathematical explanations ... then these explanations must also have a true explanans. The reason why this argument can't be used is that, in the context of an argument for realism about mathematics, it is question-begging. For we also assume here that genuine explanations must have a true explanandum, and when the explanandum is mathematical, its truth will also be in question.

Thus, for there to be something to explain at all, one assumes the truth of that which is to be explained. It is therefore immediately clear why such an effort is being made in order to find an example in the sciences. For if the explanandum contains a mathematical property, like primeness, then

if mathematical properties apply to anything, they apply prima facie to mathematical objects (Bangu, 2008, p. 18). 
Therefore, given the assumption that the explanandum is true because of the use of an inference to the best explanation, and the presence of the mathematical property of primeness in the explanandum, it would be the case that one is ontologically committed to mathematical entities, namely numbers. But then it would be the case that mathematical platonism is established beforehand, and as such the argument would indeed be circular.

\section{The response to the claim of circularity}

As Baker (2009, p. 620) rightly notices, the claim of circularity has some force. He acknowledges the fact that the way the example was initially formulated is indeed problematic. To escape the allegation of circularity, he reformulates the argument so as to prevent the explanandum from having a mathematical property, and hence from having the object(s) to which it applies. The basic structure of the argument is now presented with the explanandum no longer mentioning primeness. What is to be explained is the actual length of the life cycles in years (Baker, 2009, pp. 620-622):

\section{(D) Data:}

The length (in years) of the life cycle of cicada subspecies A is 13 .

The length (in years) of the life cycle of cicada subspecies B is 17.

The data does mention numbers however, which are of course mathematical entities, but one thing that can be done, it is suggested, is to paraphrase data (D) in firstorder logic with identity. This way, one gets rid of the reference to mathematics. So, the first datum, with $F$ as the predicate for 'year of the life cycle of cicada subspecies $\mathrm{A}^{\prime}$, in first order logic is ${ }^{2}$ :

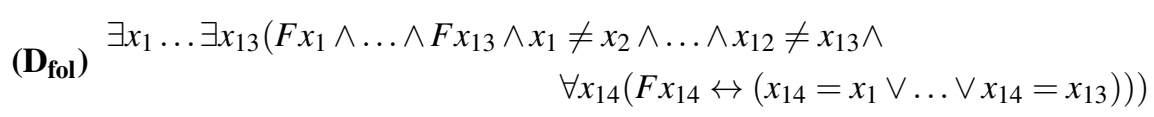

It is clear that the reason for the shift from an explanandum containing the notion of prime to an explanandum containing numbers, is that the latter can be formulated in first-order logic with identity (like above), whereas the former cannot be paraphrased away in first-order logic with identity. Baker $(2009$, p. 619) recognizes this, and refers to a proof by Boolos (1981). The reason why the notion of 'prime' in a sentence like "The length (in years) of the life cycle of cicada subspecies A is prime" cannot be expressed in first-order logic, is because of the infinite domain of prime numbers. The reference to primeness is therefore put tentatively in $(\mathrm{H})$, because it does refer to a mathematical property, and is used to ultimately 'bootstrap' the explanation of data (D) in relation to the observed primeness of the life cycles. The argument continues as follows:

\footnotetext{
${ }^{2}$ For completeness, both the 13 - as well as the 17 -year life cycles were mentioned in (D). However, to prevent too much repetition, in what follows only the first datum will be used.
} 


\section{(H) Tentative hypotheses:}

The length (in years) of the life cycle of cicada subspecies A is prime.

The length (in years) of the life cycle of cicada subspecies B is prime.

\section{(1) Biological 'law'}

Having a life cycle period which minimizes intersection with other periods is evolutionary advantageous.

\section{(2) Number theoretic theorem}

Prime periods minimize intersection (compared to non-prime periods).

\section{(3) Explanation (E)}

The lengths (in years) of the life cycles of periodical organisms are likely to be prime (explanation of $(\mathrm{H})$ ).

\section{(4) Ecological constraints}

The subspecies of Periodical Cicadas are limited by their respective biological constraints.

(5) Extended explanation $\left(\mathbf{E}^{*}\right)(\mathrm{E})$ with added biological constraints yields an explanation of (D).

One notices that steps (1) through (5) correspond to the initial argument structure given in section 1, with steps $(\mathrm{H})$ and (1) through (3) being the tentative part of the argument. The remainder of the argument is an inference to the best explanation in order to establish the ontological commitment to numbers and their properties, in this case primeness.

(6) $\left(E^{*}\right)$ is the best explanation of (D).

(7) Hence we ought to believe ( $\left.E^{*}\right)$, and thereby (E).

(8) But (D) and (E) together imply (H).

(9) Hence we ought to believe $(\mathrm{H})$

So, the basic idea underlying the reformulated argument structure is that, by paraphrasing data (D) in first-order logic with identity and putting the hypotheses pertaining to the primeness of the periodical cicadas life cycle tentatively, circularity can be prevented.

Regarding the tentative hypotheses in $(\mathrm{H})$, there are two possibilities. The primeness of the length (in years) of the life cycles could either have a common explanation, describing them as prime, or have no common explanation, in which describing them as prime is a coincidence (Baker, 2009, pp. 621-622). It is then suggested that the best explanation of tentative hypotheses $(\mathrm{H})$ is explanation (E). And, given that very fact, it is then claimed that it 
can also be turned into an explanation of ... [(D)], by adding the subspeciesspecific ecological constraints (Baker, 2009, p. 621).

This brings us to the matter of the connection between the explanandum and the explanans. How can an explanation in which the mathematical property of prime is used, such as in the mathematical explanation (E) of tentative hypothesis $(\mathrm{H})$, be turned into, or transferred to, an explanation of a datum which is paraphrased away in firstorder logic with identity, such as in $\left(\mathrm{D}_{\mathrm{fol}}\right)$ ?

\section{The circularity problem again}

The main argument of this section questions the feasibility of $\left(\mathrm{E}^{*}\right)$ yielding an explanation of (D) within the context of the Enhanced Indispensability Argument. It is noted that the inference from ' _ is (likely to be) prime', which is used in $\left(\mathrm{E}^{*}\right)$ because of (E), to ' - is 13', which is used in (D), needs explaining.

Given that the mathematical explanation of the tentative hypotheses, which states that the life cycles are prime, is accepted as the best explanation, the next step is to explain how the explanation of the tentative hypotheses can be turned into an explanation of Data (D). Recall that (D) was paraphrased in first-order logic with identity, like in $\left(D_{\text {fol }}\right)$. Note that, on the one hand, $\left(D_{\mathrm{fol}}\right)$, treats of a plurality, that is, the collection of objects expressed by the sentence in first-order logic with identity. But (E), on the other hand, treats of an entity, which (likely) has the property of being prime. This means that the context of the two sentences differs, and that therefore the two contexts need to be linked. Without such a link it is not possible to predicate primeness of a sentence in first-order logic with identity. The crucial observation is therefore that there is a gap between $\left(D_{\text {fol }}\right)$ and $\left(E^{*}\right)$ : how can the plurality of $\left(D_{\text {fol }}\right)$ end up as an entity about which $\left(\mathrm{E}^{*}\right)$ claims that it is (likely) prime?

In light of this, and thus for Baker's move to prevent circularity to work, we need some sort of bridge principle, so as to be able to logically relate the explanans to the explanandum by connecting pluralities to entities. Without such a logical connection, there is no mathematical explanation of the empirical phenomenon described by the sentence in first-order logic with identity. In light of Frege's work (Frege, 1893) and the neo-Fregean research programme (Burgess, 2005), a natural suggestion would be to use Hume's Principle (HP), which states that "for any concepts F, G, the number of $F$ is identical to the number of $G$ if and only if $F$ and $G$ are equinumerous"(Shapiro, 2000, p. 110): ${ }^{3}$

HP $\forall F \forall G(\# F=\# G \leftrightarrow F \cong G)$

The principle HP is a principle that connects the plurality of objects having properties $\mathrm{F}$ and $\mathrm{G}$ to entities (numbers). If one combines $\mathrm{HP}$ with dyadic second-order logic, then Frege's Theorem says that the Peano postulates can be deduced, given Frege's definitions of zero, successor and natural number (Burgess, 2005, p. 26). Furthermore, Dedekind's Theorem says that the existence of unique operations on natural numbers

\footnotetext{
${ }^{3}$ We follow the notation of (Boolos, 1998, p. 139)
} 
satisfying the recursion equations for addition and multiplication can be deduced in dyadic second-order logic from the Peano postulates (Burgess, 2005, p. 29). Once we have that, we can start proving results about prime numbers.

So far, so good, one might think. However, note that, given HP and Frege's definitions, $D_{\text {fol }}$ is provably equivalent with the following statement:

$\mathbf{D}_{\#} \# F=13$

Now, the question is whether this provable equivalence is also an analytical equivalence. By analytic equivalence, it is meant that the equivalence follows from nothing but logic and definitions. Since the only things that have been assumed are dyadic second-order logic, Frege's definitions and HP, the question boils down to the question whether HP is analytic.

It is clear that $\mathrm{HP}$ is at least a material equivalence statement. But neo-Fregeans will immediately point out that HP is not just a regular material equivalence. Take for instance the following example, borrowing from Quine (1953, p. 37). The material equivalence

'creature with a heart' if and only 'creature with kidneys'

does not carry over any content from the one side to the other. This is because the material equivalence does not hold out of necessity. Rather, it holds by virtue of accidental matters of fact. But HP on the other hand functions, at the very least, as an axiom for $\# F$, and is therefore, trivially, a provable equivalence. Furthermore, it is taken to be an implicit, contextual definition, thereby allowing for the cardinal numbers to be defined. As Wright (1999, p. 10) puts it:

The key idea [of HP in the neo-Fregean context] is that an instance on the left-hand side of an abstraction principle is meant to embody a reconceptualization of the type of state of affairs depicted on the right.

Because of this reconceptualization, HP is often taken to be an analytic principle.

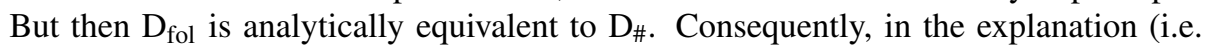
the ordered set of the explanandum and the explanans) the explanandum is analytically equivalent to a sentence that, given Frege's Theorem and Dedekind's Theorem, has to be interpreted as having mathematical content. Hence, the explanandum then also has to be interpreted as having mathematical content, but that would beg the question against the nominalist.

Boolos (1998) however, has argued that HP cannot be considered to be an analytic principle, for reasons which would complicate matters even more for a position like Baker's. The problem is that HP entails the existence of infinitely many abstract objects. And analytic principles should not commit one to so many objects, let alone abstract objects. Rather, analytic principles should be ontologically neutral (Boolos, 1998, pp. 305-306).

In response to the criticism formulated by Boolos, some have argued that questions of meaning and questions of existence should be separated. The existence of numbers does not follow from HP, or so it could be argued. Rather, they are presupposed, 
resulting from the fact that neo-Fregeans consider HP to be true. If questions of existence were indeed, pace neo-Fregeans, to be settled elsewhere, this would mean that HP would therefore not commit one to the existence of numbers, and could perhaps still be considered an analytic principle. However, this would not suffice for Baker, because it would be neutral with regard to the existence of numbers. In fact, this is why a conditional version of HP is acceptable for a nominalist (Field, 1984, p. 661):

$\mathbf{H P}_{\text {cond }}$ If numbers exist, then $\forall F \forall G(\# F=\# G \leftrightarrow F \cong G)$

Clearly, $\mathrm{HP}_{\text {cond }}$ would only bridge the gap between arithmetic on the one hand and $\mathrm{D}_{\mathrm{fol}}$ on the other hand conditional on the existence of numbers. So, we would only have a mathematical explanation if it is already agreed upon that numbers exist. But the Enhanced Indispensability Argument was supposed to justify the belief in the existence of numbers. Circularity looms again.

Wright (1999, pp. 9-10) points out, in effect, that the intelligibility of $\mathrm{HP}_{\text {cond }}$ presupposes itself that numbers exists. The antecedent 'numbers exist' will contain expressions of the form $\# X$. But there is no longer a sufficient condition to determine the truth of sentences containing those expressions and, therefore, there is no longer a contextual definition available. That is why he concludes that $\mathrm{HP}_{\text {cond }}$ has to be "relativized to an antecedently given domain of quantification" (p. 9) and that "the existence of numbers is a further fact, something which the (mere) equinumerosity of concepts may leave unresolved" (p. 10). If analyticity is supposed to be ontologically neutral, then one cannot accept the analyticity of $\mathrm{HP}_{\text {cond }}$.

This, then, is the problem the Enhanced Indispensability Argument faces when it is intended as an argument for mathematical platonism: the predicate ' _ is prime' can either not latch on to the first-order logic sentence without the latter being interpreted as a mathematical statement, resulting from the supposed analyticity of HP, or the predicate can not latch on to the first-order logic sentence with identity without making it conditional upon the existence of numbers, as is the case with a conditional version of HP. Of course, this problem depends on the supposed analyticity of HP. There is an ongoing debate about this. Recently, Boccuni and Woods (forthcoming) defend the idea that HP is a conceptual truth, whereas Darnell and Thomas-Bolduc (forthcoming) argue that HP is not analytic. We argue that the outcome of that debate is relevant for the assessment of the Enhanced Indispensability Argument. In the next section, we will investigate the option of the non-analyticity of HP.

\section{The interpretability strategy}

In the previous section a gap in the Enhanced Indispensability Argument was identified and this gap was bridged with the help of HP. It was then argued that the circularity problem resurfaces, if HP is an analytic truth. Thus, for the bootstrapping strategy as suggested by Baker to work, and to allow for the possibility to escape circularity, the Enhanced Indispensability Argument has to assume that HP is non-analytic. The nonanalyticity of HP, however, is not something which is a matter of course. This section will nonetheless suppose, for the sake of argument, that HP is not an analytic principle. 
The defender of the Enhanced Indispensability Argument could then give the following explanation: HP with dyadic second-order logic and added biological constraints yields an explanation of $\mathrm{D}_{\text {fol }}$. If this is the best explanation, then the conclusion of the indispensability argument is that one should accept the existence of (Frege-)numbers. It has not been settled yet that the mathematical explanation based on HP is the best explanation, which is needed for the Enhanced Indispensability Argument to establish platonism. This section challenges the further assumption that the Enhanced Indispensability Argument is indeed the best explanation, thereby arguing that even if it would turn out that HP could be considered a non-analytic principle, hence allaying the worries of circularity, platonism still wouldn't be established. Not only will we offer an alternative explanation strategy, we will also take up the circularity concern once more and remark that one criterion for the selection of the best explanation is question-begging.

Let us then have a look at the alternative explanation. In what follows, we assume some knowledge about intermediate logic — see, for instance, (Boolos et al, 2007). We will also make use of the notion of relative interpretation. Roughly, a relative interpretation of a theory into another theory is a translation of the language of the first theory into the language of the second theory, where the translation has to meet certain conditions, and a proof that the translation of every theorem of the first theory is a theorem of the second theory. For formal definitions, see (Enderton, 2001, section 2.7).

The starting point is a theory of concatenation, called QT (Damnjanovic, 2017, pp. 383-383). The language of QT is a first-order language with identity, individual constants $a, b$, and a binary function symbol $*$. The theory has five axioms:

$$
\begin{aligned}
& \text { QT1 }((x * y) * z)=(x *(y * z)) \\
& \text { QT2 } \neg(x * y=a) \wedge \neg(x * y=b) \\
& \text { QT3 }(x * a=y * a \rightarrow x=y) \wedge(a * x=a * y \rightarrow x=y) \wedge \\
& \qquad(x * b=y * b \rightarrow x=y) \wedge(b * x=b * y \rightarrow x=y) \\
& \text { QT4 } \neg(a * x=b * x) \wedge \neg(x * a=x * b) \\
& \text { QT5 } x=a \vee x=b \vee(\exists y(a * y=x \vee b * y=x) \wedge \exists z(z * a=x \vee z * b=x))
\end{aligned}
$$

The result is a theory about the concatenation of non-empty strings of $a$ 's and $b$ 's.

We claim that QT is an interesting starting point for a nominalist. First, Goodman and Quine (1947) made already use of expressions of concatenation in their nominalistic theory of syntax, ${ }^{4}$ which they use in an attempt to reconstruct classical mathematics. ${ }^{5}$ Second, it turns out that QT is mutually interpretable with Adjunctive Set Theory (AST) - see (Damnjanovic, 2017). The language of AST is a first-order language with binary relation symbol $\in$. The theory has only three axioms:

\footnotetext{
${ }^{4}$ In fact, any metaphysical theory that claims to be true should be compatible with a theory of truth of sentences, which depends on a theory of syntax, which in turn is hardly conceivable without some kind of theory of concatenation. See (Tarski, 1936).

${ }^{5}$ Goodman and Quine (1947, p. 106) decline to assume that there are infinitely many objects. As it will turn out, QT has no finite models. So, QT goes beyond what they are willing to assume. However, other nominalists, notably Field, have no scruples in assuming that there are infinitely many objects.
} 
NULL $\exists x \forall y \neg y \in x$

ADJ $\forall x \forall y \exists z \forall w(w \in z \leftrightarrow(w=x \vee w=z))$

EXT $\forall x \forall y(\forall z(z \in x \leftrightarrow z \in y) \rightarrow x=y)$

The result is a very weak fragment of Zermelo-Fraenkel set theory with Choice (ZFC). Field (1992) has tried to show that ZFC with so-called urelements is 'conservative' in the sense that it is consistent with any internally consistent purely physical theory. This played an important role in his nominalistic project. Since even Field thinks that ZFC with urelements is conservative, AST should definitely be so.

The good news is that QT is mutually interpretable with a very weak system of arithmetic, called Robinson Arithmetic (Q) — see (Damnjanovic, 2017). ${ }^{6}$ This is certainly interesting, but at first sight it might be disappointing that it can only interpret a very weak system of arithmetic, especially compared to second-order Peano Arithmetic $\left(\mathrm{PA}^{2}\right)$, which one can get out of the combination of HP and dyadic second-order logic. But it is important to keep the dialectic in mind. We are not in the business of giving a nominalistic reconstruction of the totality of mathematics, or even as much as is possible. We are also not preoccupied with developing a nominalistic reconstruction of all the mathematics that is used in natural science. The sole point of interest is whether there is a nominalistic explanation for those natural phenomena for which it is claimed that they are best explained mathematically.

Theory Q has some deductive power: for any $\Sigma_{1}^{0}$-sentence $\phi, \phi$ is provable in $\mathrm{Q}$ if and only if $\phi$ is true in the standard model of arithmetic. With a $\Sigma_{1}^{0}$ is meant a sentence of the form $\exists x \psi$, with $\psi$ a formula built up from atomic formulas using only the logical operators and bounded quantifiers. The latter are of the form $\forall x \leq t$ or $\exists x \leq t$ (with $t$ a term not containing $x$ ). Moreover, every recursive function and relation is definable in $\mathrm{Q}$ by a $\Sigma_{1}^{0}$-formula.

The question is now whether $\mathrm{Q}$ has enough deductive power for providing a mathematical explanation of the cicada case. First, note that the multiplication and quotient functions, the maximum and minimum functions, and the properties of being prime and co-prime belong to the categories of recursive functions and relations. We can formulate all the key notions with the help of $\Sigma_{1}^{0}$-formulas. Second, one may worry that the lemmas used by Baker are unbounded universal quantifications ranging over all numbers, not just the numbers smaller than some number. However, those unbounded universal generalisations are far stronger than are needed in the explanations of natural phenomena. Take the case of the cicadas. There is no reason to consider life cycles of cicadas that exceed, let's say, the life span of Earth. There is no problem with using bounded universal quantifications because whatever natural phenomenon that needs to be explained is going to be bounded anyway. Recall the ecological constraints that were used in the (reformulated) Enhanced Indispensability Argument. It is exceedingly

\footnotetext{
${ }^{6}$ Interestingly, Q is not interpretable in mereology, another favourite of nominalists. This is a consequence of the following theorem listed by Niebergall (2011): there is no consistent mereological theory in which AST is relatively interpretable. This is certainly relevant for the discussion about the evaluation of the nominalistic paraphrase of statements about primeness given by Tallant (2013), because he uses mereological fusions. Taking it further, he hopes that mereology will be enough to paraphrase all "scientifically explanatory mathematics". The latter cannot include Robinson Arithmetic, if his project is to succeed.
} 
likely that similar constraints will be used in any purported mathematical explanation in natural science. This strategy can also be applied to the "more general" explanation in (Baker, 2016) of the primeness of the life cycles of cicadas that is based on Bertrand's postulate.

The explanation strategy sketched above can be applied in other cases as well. The reason is that $\mathrm{Q}$ can interpret in its turn a quite wide range of mathematical theories, including (first-order) Euclidean geometry, consequence, Tarski's theory of real closed ordered fields, and the so-called Base Theory for Feasible Analysis (Ferreira and Ferreira, 2013). In all cases of explanations that essentially involve any of the mentioned theories, one can use the relative interpretability theorems to conclude, first, that there are theorems in Q that are interpretations of the theorems in the aforementioned theories and, second, that there are theorems QT that are interpretations of the relevant theorems in Q. Let us call this the interpretability strategy. Metaphorically speaking, what we propose to do is to run simulations of software written in one language within other software written in another language, and which are ultimately reducible to manipulations of bits.

Ontologically speaking, the nominalist is committed to no more than non-empty strings of $a$ 's and $b$ 's, because that is what the existential quantifiers in QT range over. ${ }^{7}$ Independently of ontological controversies, we think that the interpretability strategy is interesting in the context of debates of mathematical applications and mathematical indispensability for science. For it is worthwhile to know what the weakest mathematical theories are in which one can interpret whatever mathematics is used in scientific explanations. $^{8}$

There are at least two alternative explanations available in the cicada case. The first is an explanation invoking Hume's Principle (considered to be non-analytic), dyadic second-order logic and some ecological constraints. The second is an explanation invoking Robinson Arithmetic, which is subsequently interpreted within a theory of concatenation of non-empty strings of $a$ 's and $b$ 's. If one wants to conclude that (Frege)numbers exist, which is in line with platonism and which goes against nominalism, it needs to be established in that the first explanation is the best. This has to happen without begging the question. Frege Arithmetic has a lot more mathematical strength than Robinson Arithmetic. But a nominalist should not be concerned about that, whereas a difference in empirical scope would be cause for concern. This is a challenge for the defender of the Enhanced Indispensability Argument.

\footnotetext{
${ }^{7}$ We are relying here on Quine's criterion for ontological commitment (Quine, 1948). Some participants in the debate have questioned this criterion — see, for example, Azzouni (2004).

${ }^{8}$ Our strategy is in this sense similar to a strategy suggested by Bueno (2001, p. 119). His idea was for a nominalist to capitalize on the so-called reverse mathematics programme, initiated by Harvey Friedman, and aiming at discovering what the weakest axioms are to prove a theorem. There are a couple of differences though. One is that Bueno is aiming at a nominalistic reconstruction of as much of mathematics as possible, whereas we are only talking about what Tallant (2013, p. 2074) calls "scientifically explanatory mathematics". Another difference is that Bueno focuses on abstraction principles, which he wants to paraphrase using a combination of plural logic and mereology.
} 


\section{Conclusion}

The much discussed case study of the prime numbered life cycle of the Periodical Cicada, as presented by Baker $(2005,2009)$, presents an interesting account in which mathematics (and, according to Baker (2017a), even sophisticated mathematics) appears to play an indispensable role in the explanation of an empirical phenomenon. The question this paper set out to answer was whether it can be used as an ontological argument for mathematical platonism.

For there to be a mathematical explanation of the first-order logic datum at all, the mathematical explanans needs to be able to connect to the explanandum. However, resulting from the strategy to paraphrase the reference to numbers away in first-order logic with identity, the explanandum now treats of a plurality. This means that the context differs in the two cases. Without a link between the two contexts, it is not possible to predicate primeness of the sentence in first-order logic with identity. To overcome the gap, Hume's Principle is a natural suggestion to function as a bridge principle, because it can be used in combination with dyadic second-order logic to say things about prime numbers. This is relevant, because without it there would be no logical connection between the plurality in the explanandum and the property of primeness in the explanans, and thereby no mathematical explanation. Several philosophers think that Hume's Principle is an analytic truth. But then the explanandum has therefore, inevitably, to be interpreted as having mathematical content (since it is analytically equivalent to a mathematical sentence). And for this reason the argument ultimately begs the question against the mathematical nominalist.

Some philosophers think that Hume's Principle is not an analytic truth. In that case there can be a mathematical explanation of the explanandum, paraphrased in firstorder logic. This explanation makes use of Hume's Principle, dyadic second-order logic, which together form a theory called 'Frege Arithmetic', and certain ecological constraints. It is, however, not settled that this is the best explanation. In order to provide an alternative explanation we develop the so-called interpretability strategy. The idea is to use the relative interpretability of several mathematical theories into an elementary theory of arithmetic, called 'Robinson Arithmetic', which is in turn interpretable in a theory of concatenation of non-empty strings of $a$ 's and $b$ 's. Acceptance of the latter theory only commits one to the existence of those strings, but at the same time one can prove the interpreted theorems of quite a bit of mathematics. Sure, the mathematical power of Robinson Arithmetic is much less than the mathematical power of Frege Arithmetic, but for a nominalist that is a question-begging difference, whereas a difference in empirical scope would not be question-begging.

Regardless of the question whether mathematics does indeed do (part of) the explanatory work in the discussed example, two take-home lessons are the following. First, the debate on the status of Hume's Principle as an analytic truth is relevant for the debate on the Enhanced Indispensability Argument. More generally, this applies to the debate on the status of so-called abstraction principles as well. Second, the logical and mathematical work on relative interpretations of mathematical theories should be of great interest for anyone working on the topics of mathematical applications and mathematical indispensability. 
Acknowledgements We would like to thank two anonymous reviewers for their very helpful comments. Earlier versions of this paper have been presented at the ParisLeuven Workshop (Paris, 11 October 2018), the IX Conference of the Spanish Society for Logic, Methodology and Philosophy of Science (Madrid, 13 Nov 2018-16 Nov 2018), the seminar of the Philosophy department in Stellenbosch (Stellenbosch, 7 December 2019) and the CLPS Seminar (Leuven, 1 March 2019). We would like to thank all the audiences for their useful feedback. Finally, we would also like to thank Leon Horsten for discussing the topic of this paper with one of the authors.

\section{References}

Azzouni J (2004) Deflating Existential Commitment: A Case for Nominalism. Oxford University Press

Baker A (2005) Are there genuine mathematical explanations of physical phenomena? Mind 114(454):223-238

Baker A (2009) Mathematical explanation in science. British Journal for the Philosophy of Science 60(3):611-633

Baker A (2016) Parsimony and inference to the best mathematical explanation. Synthese 193(2)

Baker A (2017a) Mathematical spandrels. Australasian Journal of Philosophy 95(4):779-793

Baker A (2017b) Mathematics and explanatory generality. Philosophia Mathematica 25(2):194-209

Bangu SI (2008) Inference to the best explanation and mathematical realism. Synthese 160(1):13-20

Barrantes M (2019) Optimal representations and the enhanced indispensability argument. Synthese 196(1):247-263

Boccuni F, Woods J (forthcoming) Structuralist neologicism. Philosophia Mathematica

Boolos G (1981) For every A there is a B. Linguistic Inquiry 12(3):465-467

Boolos G (1998) Logic, Logic, and Logic. Harvard University Press

Boolos G, Burgess J, P R, Jeffrey C (2007) Computability and Logic. Cambridge University Press

Bueno O (2001) Logicism revisited. Principia 5(1-2):99-124

Burgess JP (2005) Fixing Frege. Princeton University Press

Colyvan M (2002) Mathematics and aesthetic considerations in science. Mind 111(441):69-74 
Damnjanovic Z (2017) Mutual interpretability of Robinson Arithmetic and Adjunctive Set Theory with Extensionality. Bulletin of Symbolic Logic 23(4):381-404

Darnell E, Thomas-Bolduc A (forthcoming) Is Hume's principle analytic? Synthese pp $1-17$

Enderton HB (2001) A Mathematical Introduction to Logic. Academic Press

Ferreira F, Ferreira G (2013) Interpretability in Robinson's Q. Bulletin of Symbolic Logic 19(3):289-317

Field H (1984) Frege's conception of numbers as objects. Canadian Journal of Philosophy 14(4):637-662

Field H (1989) Realism, Mathematics and Modality. Basil Blackwell ltd.

Field H (1992) A nominalistic proof of the conservativeness of set theory. Journal of Philosophical Logic 21(2):111-123

Frege G (1893) Grundgesetze der Arithmetik, Begriffsschriftlich abgeleitet, vol 1. Verlag Hermann Pohle, Jena

Goodman N, Quine WV (1947) Steps toward a constructive nominalism. Journal of Symbolic Logic 12(4):105-122

Leng M (2005) Mathematical explanation. In: Cellucci C, Gillies D (eds) Mathematical Reasoning, Heuristics and the development of mathematics, London: King's College Publications, pp 167-189

Maddy P (1992) Indispensability and practice. Journal of Philosophy 89(6):275-289

Melia J (2000) Weaseling away the indispensability argument. Mind 109(435):455480

Niebergall KG (2011) Mereology. In: Horsten L, Pettigrew R (eds) The Continuum Companion to Philosophical Logic, pp 271-298

Putnam H (1971) Philosophy of Logic. London: Allen \& Unwin

Quine WV (1948) On what there is. Review of Metaphysics 2(1):21-38

Quine WV (1953) From a Logical Point of View. Harvard University Press

Rizza D (2011) Magicicada, mathematical explanation and mathematical realism. Erkenntnis 74:101-114

Shapiro S (2000) Thinking about mathematics: the philosophy of mathematics. Oxford University Press

Tallant J (2013) Optimus Prime: Paraphrasing prime number talk. Synthese 190(12):2065-2083 
Tarski A (1936) The concept of truth in formalized languages. In: Tarski A (ed) Logic, Semantics, Metamathematics, Oxford University Press, pp 152-278

Wakil S, Justus J (2017) Mathematical explanation and the biological optimality fallacy. Philosophy of Science 84:916-930

Wright C (1999) Is Hume's principle analytic? Notre Dame Journal of Formal Logic 40(1):6-30 